PROCEEDINGS OF THE

AMERICAN MATHEMATICAL SOCIETY

Volume 129, Number 5, Pages 1395-1401

S 0002-9939(00)05722-1

Article electronically published on October 25, 2000

\title{
STRICHARTZ ESTIMATES FOR THE SCHRÖDINGER EQUATION WITH RADIAL DATA
}

\author{
ATANAS STEFANOV
}

(Communicated by Christopher D. Sogge)

\begin{abstract}
We prove an endpoint Strichartz estimate for radial solutions of the two-dimensional Schrödinger equation:

$$
\left\|e^{-i t \Delta} f\right\|_{L_{t}^{2} B M O_{x}} \leq C\|f\|_{L^{2}} .
$$
\end{abstract}

\section{INTRODUCTION}

We consider the inhomogeneous initial value problem for the Schrödinger equation

$$
\begin{cases}\partial_{t} u(x, t), & =-i \Delta_{x} u+F(x, t), \quad(x, t) \in \mathbf{R}^{n} \times \mathbf{R}^{1}, \\ u(x, 0) & =f(x) .\end{cases}
$$

We introduce the operator $e^{-i t \Delta}$ as

$$
e^{-i t \Delta} f=\mathcal{F}^{-1}\left(e^{i t|\cdot|^{2}} \hat{f}(\cdot)\right),
$$

where $\mathcal{F}, \mathcal{F}^{-1}$ are the Fourier transform and the inverse Fourier transform respectively. The solution to (1) is given by $u(x, t)=e^{-i t \Delta} f+e^{-i t \Delta} \int_{0}^{t} e^{i \tau \Delta} F(\tau, \cdot) d \tau$, at least for sufficiently "good" data $f, F$. A lot of attention has been given to the problem of determining all possible Strichartz estimates for the solutions of the Schrödinger equation. To state the known results, we use the following mixed space notation:

$$
\|u\|_{L_{t}^{q} L_{x}^{r}}:=\left(\int_{\mathbf{R}^{1}}\left(\int_{\mathbf{R}^{n}}|u(x, t)|^{r} d x\right)^{q / r} d t\right)^{1 / q} .
$$

In addition, we call a pair $(q, r)$ Schrödinger admissible if $q, r \geq 2,(q, r, n / 2) \neq$ $(2, \infty, 1), 2 / q+n / r=n / 2$, where $n$ stands for the spatial dimension. Then

$$
\begin{aligned}
\left\|e^{-i t \Delta} f\right\|_{L_{t}^{q} L_{x}^{r}} & \leq C\|f\|_{L^{2}}, \\
\left\|e^{-i t \Delta} \int_{0}^{t} e^{i \tau \Delta} F(\tau, \cdot) d \tau\right\|_{L_{t}^{\tilde{q}} L_{x}^{\tilde{r}}} & \leq C\|F\|_{L_{t}^{q^{\prime}} L_{x}^{r^{\prime}}}
\end{aligned}
$$

Received by the editors August 6, 1999.

1991 Mathematics Subject Classification. Primary 35J10; Secondary 42B15.

Key words and phrases. Schrödinger equation, Strichartz estimates.

This research was supported in part by DMS-9870027. 
for all Schrödinger admissible pairs $(q, r),(\tilde{q}, \tilde{r})$. Some earlier versions of the results above appeared in [7], 11], [1], whereas the final (endpoint) estimate for dimension $n \geq 3$ was proved recently in [2]. One can easily see that homogeneity dictates the condition $2 / q+n / r=n / 2$, while the restriction $q, r \geq 2$ comes from a concrete counterexample due to Knapp and properties of translation invariant operators (see [2]). The point $q=2, r=\infty$ in the two-dimensional case is excluded from the range of admissibility, because the estimate there is no longer valid by a counterexample due to Montgomery-Smith [5]. He actually proved something more:

$$
\sup _{\|f\|_{L^{2}}=1}\left\|e^{-i t \Delta f}\right\|_{L_{t}^{2} B M O_{x}}=\infty
$$

Some further generalization of the ideas in [5] provided counterexamples for some similar conjectures, concerning frequency localized and BMO estimates in both the Schrödinger and the wave equation case; see [9] for details. Let us remark that all of the aforementioned results have their counterparts for the solutions of the wave equation with slightly different admissibility conditions. In [10], one can find weighted inequalities for the endpoint case with applications to radial solutions, but that is only in dimension $n \geq 3$. Our main goal is to show that the "modified" Strichartz estimate in the critical case $q=2, r=\infty, n=2$ remains valid as long as we restrict our attention to radial data 1

Thus, the theorem below is the main result of this paper.

Theorem 1. Let $f$ be a radial function. Then

$$
\left\|e^{-i t \Delta} f\right\|_{L_{t}^{2} B M O_{x}} \leq C\|f\|_{L^{2}}, \quad x \in \mathbf{R}^{2}
$$

where BMO is the space of functions with bounded mean oscillation.

Remark 1. For the wave equation a similar theorem in the wave critical case $q=$ $2, r=\infty, n=3$ was established by Klainerman and Machedon [3].

\section{Preliminary Steps AND NOTATIONS}

In this section we make several technical reductions of the problem. Introduce first $\varphi \in C_{0}^{\infty}\left(\mathbf{R}^{1}\right)$ supported in $(1 / 2,2), \varphi(x)=1$ for $x \in(3 / 4,9 / 8)$ and $\sum_{k \in \mathbf{Z}} \varphi\left(2^{-k} t\right)=1$ for all $t>0$. Let $\varphi_{k}(t)=\varphi\left(2^{-k} t\right)$. We can define a LittlewoodPaley decomposition with respect to $\left\{\varphi_{k}\right\}$ by setting $P_{k} f=\mathcal{F}^{-1}\left(\hat{f}(\cdot) \varphi_{k}(|\cdot|)\right)$. It is a standard fact that

$$
\|g\|_{B M O} \sim\left\|\left(\sum_{k \in \mathbf{Z}}\left|P_{k} g\right|^{2}\right)^{1 / 2}\right\|_{L_{\infty}} .
$$

Therefore, the theorem would follow from the following localized version:

$$
\left\|e^{-i t \Delta} P_{k} f\right\|_{L_{t}^{2} L_{x}^{\infty}} \lesssim\|f\|_{L^{2}} .
$$

\footnotetext{
${ }^{1}$ The author has been informed that T. Tao has an independent proof of the original Strichartz estimate in the radial case.
} 
Indeed, let $\psi$ be a smooth function with support contained in $(1 / 4,4)$ and $\psi(x) \varphi(x)$ $=\varphi(x)$. Then

$$
\begin{aligned}
& \left\|e^{-i t \Delta} f\right\|_{L_{t}^{2} B M O_{x}}^{2} \lesssim \int \sup _{x}\left(\sum_{k}\left|e^{-i t \Delta} P_{k} f\right|^{2}\right) d t \\
& \lesssim \sum_{k}\left\|e^{-i t \Delta} P_{k} \tilde{P}_{k} f\right\|_{L_{t}^{2} L_{x}^{\infty}}^{2} \lesssim \sum_{k}\left\|\tilde{P}_{k} f\right\|_{L^{2}}^{2} \lesssim\|f\|_{L^{2}}^{2},
\end{aligned}
$$

where $\tilde{P}_{k} f=\mathcal{F}^{-1}\left(\hat{f} \psi_{k}\right)$.

Let $f_{0}(\rho):=\hat{f}(\xi)$ for $|\xi|=\rho$. Write

$$
\begin{aligned}
& \left\|e^{-i t \Delta} P_{k} f\right\|_{L_{t}^{2} L_{x}^{\infty}}^{2}=\frac{1}{2 \pi} \int \sup _{x}\left|\int_{\mathbf{R}^{2}} e^{i t|\xi|^{2}} e^{i\langle\xi, x\rangle} \hat{f}(\xi) \varphi\left(2^{-k}|\xi|\right) d \xi\right|^{2} d t \\
& =\frac{1}{2 \pi \Gamma(1 / 2)} \int \sup _{x}\left|\int_{0}^{\infty} \int_{-1}^{1} e^{i t \rho^{2}} e^{i|x| \rho \sigma}\left(1-\sigma^{2}\right)^{-1 / 2} f_{0}(\rho) \varphi\left(2^{-k} \rho\right) \rho d \sigma d \rho\right|^{2} d t .
\end{aligned}
$$

Next, linearize in $|x|(|x|=r(t)$ is the value of $|x|$ for which the sup is "achieved") and change variables $\rho \rightarrow \sqrt{\rho}$ to write the desired inequality as:

$$
\int\left|\int_{0}^{\infty} \int_{-1}^{1} e^{i t \rho} e^{i r(t) \sqrt{\rho} \sigma} f_{0}(\sqrt{\rho}) \varphi\left(2^{-k} \sqrt{\rho}\right)\left(1-\sigma^{2}\right)^{-1 / 2} d \sigma d \rho\right|^{2} d t \lesssim \int_{\mathbf{R}^{2}}|f(x)|^{2} d x .
$$

Since $\int_{\mathbf{R}^{2}}|f(x)|^{2} d x \sim \int_{0}^{\infty}\left|f_{0}(\sqrt{\rho})\right|^{2} d \rho$, it suffices to prove

$$
\int\left|\int_{0}^{\infty} \int_{-1}^{1} e^{i t \rho} e^{i r(t) \sqrt{\rho} \sigma} g(\rho) \varphi\left(2^{-k} \sqrt{\rho}\right)\left(1-\sigma^{2}\right)^{-1 / 2} d \sigma d \rho\right|^{2} d t \lesssim \int|g(\rho)|^{2} d \rho,
$$

where $r(t)$ is an arbitrary positive measurable function and $k$ is a fixed integer. By homogeneity, it suffices to prove the above estimate for $k=0$. Denote $A_{j}=\{t$ : $\left.2^{j-1} \leq r(t)<2^{j}\right\}$. We need to prove

$$
\sum_{j} \int_{A_{j}}\left|\int_{-1}^{1} \int e^{i t \rho} e^{i r(t) \sqrt{\rho} \sigma} g(\rho) \varphi(\rho)\left(1-\sigma^{2}\right)^{-1 / 2} d \rho d \sigma\right|^{2} d t \lesssim\|g\|_{2}^{2} .
$$

In the sequel, we will need the following well-known lemma concerning a cutoff version of the Bessel function $J_{0}$ that appears above.

Lemma 1. Let $p \geq 0$ and $M, \kappa \geq 1$. Then

$$
\begin{aligned}
& \left|\int_{-1}^{1} e^{i \kappa \sigma}\left(1-\sigma^{2}\right)^{-1 / 2} \varphi\left(2^{p}\left(1-\sigma^{2}\right)\right) d \sigma\right| \leq C_{M} \min \left\{\left(2^{p} /|\kappa|\right)^{M} 2^{-p / 2}, 2^{-p / 2}\right\}, \\
& \left|\int_{-1}^{1} e^{i \kappa \sigma}\left(1-\sigma^{2}\right)^{-1 / 2} d \sigma\right| \lesssim|\kappa|^{-1 / 2} .
\end{aligned}
$$


Since (5) follows from a simple integration by parts argument and (6) is a corollary of (5), we omit the proof. Let us remark that the "critical" index $p_{0}$ is the one for which $2^{p_{0}} \sim|\kappa|$. If $p>p_{0}$ one estimates by putting absolute value inside the integral, while if $p<p_{0}$ one integrates by parts and then estimates by absolute value. We use this idea repeatedly throughout the proof. Observe that for $p=0$, we have

$$
\left|\int_{-1}^{1} e^{i \kappa \sigma}\left(1-\sigma^{2}\right)^{-1 / 2} \varphi\left(\left(1-\sigma^{2}\right)\right) d \sigma\right| \leq C_{M}|\kappa|^{-M}
$$

\section{Main estimates}

In this section, we obtain pointwise estimates by the Hardy-Littlewood maximal function if we have enough oscillation in the term $e^{i r(t) \sqrt{\rho} \sigma}$ and we use an almost orthogonality argument otherwise. Thus, we consider the following cases.

First, $j \leq 0$. Then $|r(t) \sqrt{\rho} \sigma| \leq 2^{j+2} \leq 4$, for $r(t) \sim 2^{j}, \sqrt{\rho} \sim 1$. Therefore we have virtually no oscillation in the term $e^{i r(t) \sqrt{\rho} \sigma}$. Expand in a Maclaurin series to get

$$
\begin{aligned}
& \sum_{j \leq 0} \int_{A_{j}}\left|\int_{-1}^{1} \int e^{i t \rho} e^{i r(t) \sqrt{\rho} \sigma} g(\rho) \varphi(\rho)\left(1-\sigma^{2}\right)^{-1 / 2} d \rho d \sigma\right|^{2} d t \\
= & \sum_{j \leq 0} \int_{A_{j}}\left|\sum_{l=0}^{\infty} \frac{(i r(t))^{l}}{l !} \int e^{i t \rho} g(\rho) \varphi(\rho) \rho^{l / 2} d \rho \int_{-1}^{1}\left(1-\sigma^{2}\right)^{-1 / 2} \sigma^{l} d \sigma\right|^{2} d t .
\end{aligned}
$$

Observe that $\left|\int_{-1}^{1}\left(1-\sigma^{2}\right)^{-1 / 2} \sigma^{l} d \sigma\right| \lesssim 1$ and we have an exact inverse Fourier transform in each term above. Thus, for $l=0$ we bound by $C\|g\|_{2}^{2}$, whereas for $l \geq 1$, we estimate by

$$
\begin{gathered}
C \sum_{l \geq 1} \sum_{j \leq 0} \int_{A_{j}} \frac{r(t)^{2 l}}{l !}\left|\int e^{i t \rho} g(\rho) \varphi(\rho) \rho^{l / 2} d \rho\right|^{2} d t \\
\lesssim \sum_{l \geq 1} \sum_{j \leq 0} \frac{2^{2 j l}}{l !} \int|g(\rho)|^{2} \varphi(\rho) \rho^{l} d \rho \lesssim \sum_{l \geq 1} \sum_{j \leq 0} \frac{2^{2(j+2) l}}{l !}\|g\|_{2}^{2} \lesssim\|g\|_{2}^{2} .
\end{gathered}
$$

This completes the proof in the case $j \leq 0$.

Let us consider the other case $j \geq 1$. We have to prove

$$
\sum_{j \geq 1} \int_{A_{j}}\left|\int_{-1}^{1} \int e^{i t \rho} e^{i r(t) \sqrt{\rho} \sigma} g(\rho) \varphi(\rho)\left(1-\sigma^{2}\right)^{-1 / 2} d \rho d \sigma\right|^{2} d t \lesssim\|g\|_{2}^{2} .
$$

By Plancherel's identity, we get

$$
\begin{aligned}
& \int_{-1}^{1}\left(1-\sigma^{2}\right)^{-1 / 2}\left(\int e^{i t \rho} g(\rho) \varphi(\rho) e^{i r(t) \sqrt{\rho} \sigma} d \rho\right) d \sigma \\
= & \frac{1}{2 \pi} \int_{-1}^{1}\left(1-\sigma^{2}\right)^{-1 / 2} \int \hat{g}(y-t)\left(\int e^{i \rho y} e^{i r(t) \sqrt{\rho} \sigma} \varphi(\rho) d \rho\right) d y d \sigma .
\end{aligned}
$$

It is obvious now that (7) would follow from the following pointwise estimate. 
Proposition 1. Let $r(t)$ be a measurable function, $2^{j-1} \leq r(t)<2^{j}$. Then

$$
\int h(y-t)\left|\int_{-1}^{1}\left(1-\sigma^{2}\right)^{-1 / 2}\left(\int e^{i\left(\rho^{2} y+r(t) \rho \sigma\right)} \varphi(\rho) \rho d \rho\right) d \sigma\right| d y \lesssim M(h)(-t),
$$

where $M$ is the Hardy-Littlewood maximal operator.

Proof. Let us split the $y$ integration in three parts: $|y| \leq 2^{j} / 10,2^{j} / 10 \leq|y| \leq$ $102^{j},|y| \geq 102^{j}$.

For $|y| \leq 2^{j} / 10$, set $1=\varphi\left(1-\sigma^{2}\right)+\left(1-\varphi\left(1-\sigma^{2}\right)\right)$. If $|\sigma| \ll 1$, we use Lemma 1 with $p=0$ to estimate

$$
\left|\int_{-1}^{1} e^{i r(t) \rho \sigma}\left(1-\sigma^{2}\right)^{-1 / 2} \varphi\left(1-\sigma^{2}\right) d \sigma\right| \lesssim 2^{-j} .
$$

If $|\sigma| \sim 1$ (i.e. $\left(1-\varphi\left(1-\sigma^{2}\right)\right) \neq 0$ ), we have that $\left|\left(\rho^{2} y+r(t) \rho \sigma\right)^{\prime}\right|=|2 \rho y+r(t) \sigma| \sim 2^{j}$ and therefore we can integrate in parts in $\rho$ to obtain

$$
\left|\int e^{i\left(\rho^{2} y+r(t) \rho \sigma\right)} \varphi(\rho) \rho d \rho\right| \lesssim 2^{-j}
$$

In either case, we have

$$
\begin{aligned}
& \left|\int_{-1}^{1}\left(1-\sigma^{2}\right)^{-1 / 2} \int_{|y| \leq 2^{j} / 10} h(y-t)\left(\int e^{i\left(\rho^{2} y+r(t) \rho \sigma\right)} \varphi(\rho) \rho d \rho\right) d y d \sigma\right| \\
& \quad \lesssim 2^{-j} \int_{|y| \leq 2^{j} / 10}|h(y-t)| d y \lesssim M(h)(-t) .
\end{aligned}
$$

For $|y| \geq 102^{j}$, we have $\left|\left(\rho^{2} y+r(t) \rho \sigma\right)^{\prime}\right|=|2 \rho y+r(t) \sigma| \sim|y|$ and therefore integration by parts in $\rho$ gives

$$
\left|\int e^{i\left(\rho^{2} y+r(t) \rho \sigma\right)} \varphi(\rho) \rho d \rho\right| \lesssim|y|^{-2} .
$$

Therefore,

$$
\begin{aligned}
& \left|\int_{-1}^{1}\left(1-\sigma^{2}\right)^{-1 / 2} \int_{|y| \geq 102^{j}} h(y-t)\left(\int e^{i\left(\rho^{2} y+r(t) \rho \sigma\right)} \varphi(\rho) \rho d \rho\right) d y d \sigma\right| \\
& \quad \lesssim \int_{|y| \geq 102^{j}} \frac{|h(y-t)|}{|y|^{2}} d y \lesssim M(h)(-t) .
\end{aligned}
$$

Finally, if $2^{j} / 10 \leq|y| \leq 102^{j}$, it will suffice to prove the inequality

$$
\left|\int_{-1}^{1}\left(1-\sigma^{2}\right)^{-1 / 2} \int e^{\left(i \rho^{2} y+i r(t) \rho \sigma\right)} \varphi(\rho) \rho d \rho d \sigma\right| \lesssim 2^{-j}
$$

To show that, we have to exploit the oscillation in both $\sigma$ and $\rho$. Write

$$
\begin{aligned}
& \left|\int_{-1}^{1}\left(1-\sigma^{2}\right)^{-1 / 2} \int e^{\left(i \rho^{2} y+i r(t) \rho \sigma\right)} \varphi(\rho) \rho d \rho d \sigma\right| \\
& \quad \lesssim \sum_{p \geq 0}\left|\int_{-1}^{1}\left(1-\sigma^{2}\right)^{-1 / 2} \varphi\left(2^{p}\left(1-\sigma^{2}\right)\right) \int e^{i\left(\rho^{2} y+r(t) \rho \sigma\right)} \varphi(\rho) \rho d \rho d \sigma\right|
\end{aligned}
$$


For $p \geq j$ ( $j$ is the critical index as discussed in Lemma 1$)$, we have

$$
\left|\int_{-1}^{1}\left(1-\sigma^{2}\right)^{-1 / 2} \varphi\left(2^{p}\left(1-\sigma^{2}\right)\right) d \sigma\right| \lesssim 2^{-p / 2}
$$

and

$$
\begin{aligned}
& \left|\int e^{i\left(\rho^{2} y+r(t) \rho \sigma\right)} \varphi(\rho) \rho d \rho\right|=\left|\int e^{i y(\rho+r(t) \sigma / 2 y)^{2}} \varphi(\rho) \rho d \rho\right| \\
& \quad \lesssim\left|\int_{|\rho+r(t) \sigma / 2 y| \leq 2^{-j / 2}} e^{i y(\rho+r(t) \sigma / 2 y)^{2}} \varphi(\rho) \rho d \rho\right| \\
& \quad+\sum_{l \geq 0}\left|\int e^{i y(\rho+r(t) \sigma / 2 y)^{2}} \varphi\left(2^{-l+j / 2}(\rho+r(t) \sigma / 2 y)\right) \varphi(\rho) \rho d \rho\right| \\
& \quad \lesssim 2^{-j / 2}+\sum_{l \geq 0}\left|\int \frac{\varphi(\rho)}{y(\rho+r(t) \sigma / 2 y)} \varphi\left(2^{-l+j / 2}(\rho+r(t) \sigma / 2 y)\right) \rho d e^{i y(\rho+r(t) \sigma / 2 y)^{2}}\right| .
\end{aligned}
$$

Let us integrate by parts now in the $l^{\text {th }}$ term. We get

$$
\begin{aligned}
& \left|\int \frac{\varphi(\rho) \rho}{y(\rho+r(t) \sigma / 2 y)} \varphi\left(2^{-l+j / 2}(\rho+r(t) \sigma / 2 y)\right) d e^{i y(\rho+r(t) \sigma / 2 y)^{2}}\right| \\
& \quad \lesssim\left|\int \frac{\varphi(\rho) \rho}{y(\rho+r(t) \sigma / 2 y)^{2}} \varphi\left(2^{-l+j / 2}(\rho+r(t) \sigma / 2 y)\right) e^{i y(\rho+r(t) \sigma / 2 y)^{2}} d \rho\right| \\
& \quad+2^{-l+j / 2}\left|\int \frac{\varphi(\rho) \rho}{y(\rho+r(t) \sigma / 2 y)} \varphi^{\prime}\left(2^{-l+j / 2}(\rho+r(t) \sigma / 2 y)\right) e^{i y(\rho+r(t) \sigma / 2 y)^{2}} d \rho\right| \\
& \quad+\left|\int \frac{(\rho \varphi(\rho))^{\prime}}{y(\rho+r(t) \sigma / 2 y)} \varphi\left(2^{-l+j / 2}(\rho+r(t) \sigma / 2 y)\right) e^{i y(\rho+r(t) \sigma / 2 y)^{2}} d \rho\right| .
\end{aligned}
$$

We easily estimate the first and the second integrals above by $2^{-j / 2-l}$ and after one more integration by parts in the third one, we obtain

$$
\begin{aligned}
& \left|\int \frac{(\rho \varphi(\rho))^{\prime}}{y(\rho+r(t) \sigma / 2 y)} \varphi\left(2^{-l+j / 2}\left(\rho+\frac{r(t) \sigma}{2 y}\right)\right) e^{i y(\rho+r(t) \sigma / 2 y)^{2}} d \rho\right| \\
& \lesssim 2^{-j}\left(2^{-2 l}+2^{-3 l} 2^{j / 2}\right) .
\end{aligned}
$$

Going back to (10), we conclude

$$
\left|\int e^{i\left(\rho^{2} y+r(t) \rho \sigma\right)} \varphi(\rho) \rho d \rho\right| \lesssim 2^{-j / 2} .
$$

Therefore by combining (9) and (12), one gets

$$
\begin{aligned}
& \sum_{p \geq j}\left|\int_{-1}^{1}\left(1-\sigma^{2}\right)^{-1 / 2} \varphi\left(2^{p}\left(1-\sigma^{2}\right)\right) \int e^{\left(i \rho^{2} y+i r(t) \rho \sigma\right)} \varphi(\rho) \rho d \rho d \sigma\right| \\
& \quad \lesssim \sum_{p \geq j} 2^{-p / 2} 2^{-j / 2} \lesssim 2^{-j}
\end{aligned}
$$

which completes the proof for $p \geq j$. 
For $p \leq j$, we first integrate by parts with respect to $\sigma$ to get

$$
\begin{aligned}
\int_{-1}^{1}( & \left.1-\sigma^{2}\right)^{-1 / 2} \varphi\left(2^{p}\left(1-\sigma^{2}\right)\right) e^{i r(t) \rho \sigma} d \sigma \\
& =-\int_{-1}^{1} \frac{\left[\left(1-\sigma^{2}\right)^{-1 / 2} \varphi\left(2^{p}\left(1-\sigma^{2}\right)\right)\right]^{\prime}}{i r(t) \rho} e^{i r(t) \rho \sigma} d \sigma \\
& =\int_{-1}^{1} 2 \sigma\left(1-\sigma^{2}\right)^{-1 / 2} 2^{p} \frac{\varphi^{\prime}\left(2^{p}\left(1-\sigma^{2}\right)\right)}{i r(t) \rho} e^{i r(t) \rho \sigma} d \sigma+\text { a similar term. }
\end{aligned}
$$

We estimate

$$
\left|\int_{-1}^{1} 2 \sigma\left(1-\sigma^{2}\right)^{-1 / 2} 2^{p} \frac{\varphi^{\prime}\left(2^{p}\left(1-\sigma^{2}\right)\right)}{i r(t)} d \sigma\right| \lesssim 2^{p / 2} 2^{-j}
$$

and a repetition of the argument leading to (12) gives

$$
\left|\int e^{i\left(\rho^{2} y+r(t) \rho \sigma\right)} \varphi(\rho) d \rho\right| \lesssim 2^{-j / 2}
$$

Summing over all $p \leq j$ yields the desired bound $C 2^{-j}$.

\section{REFERENCES}

[1] J. Ginibre and G. Velo, Smoothing Properties and Retarded estimates for Some Dispersive Evolution Equations, Comm. Math. Phys. 123 (1989), 535-573.

[2] M. Keel and T. Tao, Endpoint Strichartz Estimates, Amer. J. Math. 120 (1998), 955-980. CMP 99:01

[3] S. Klainerman and M. Machedon, Space-time estimates for null forms and the local existence theorem, Comm. Pure Appl. Math. 46 (1993), 1221-1268. MR 94h:35137

[4] G. Mockenhaupt, A. Seeger, C.D. Sogge, Local Smoothing of Fourier Integrals and CarlesonSjölin Estimates, J. Amer. Math. Soc. 6 (1993), 65-130. [MR 93h:58150

[5] S.J. Montgomery-Smith, Time Decay for the Bounded Mean Oscillation of Solutions of the Schrödinger and Wave Equations, Duke Math. J. 91 (1998), no. 2, 393-408. MR 99e:35006

[6] D. Müller, A. Seeger, Inequalities for Spherically Symmetric Solutions of the Wave Equation, Math. Z. 218 (1995), 417-426. MR 96e:35093

[7] R.S. Strichartz, Restriction of Fourier Transform to Quadratic Surfaces and Decay of Solutions of Wave Equations, Duke Math. J. 44, (1977), 705-714. MR 58:23577

[8] E. M. Stein, Harmonic analysis: Real variable methods, orthogonality, and oscillatory integrals, Princeton University Press, Princeton NJ, 1993. MR 95c:42002

[9] T. Tao, Counterexamples to the $n=3$ Endpoint Strichartz estimate for the wave equation, (1998), preprint.

[10] M.C. Vilela, Regularity for the solutions to the free Schrödinger equation with radial initial data, preprint.

[11] K. Yajima, Existence of solutions for Schrödinger evolution equations, Comm. Math. Phys. 110 (1987), 415-426. MR 88e:35048

Department of Mathematics, Syracuse University, Syracuse, New York 13244

E-mail address: astefano@syr.edu 